\title{
ON COLLINEATIONS OF SYMMETRIC DESIGNS
}

\author{
E. T. PARKER ${ }^{1}$
}

This note is concerned with systems known as symmetric balanced incomplete block designs, or $(v, k, \lambda)$ configurations. (See [1] for background and bibliography.) Following the terminology of projective geometry, the elements and the distinguished subsets will be called points and lines respectively. A collineation is a permutation of the points which preserves the class of lines without regard to order of points within lines.

First will be established:

Theorem 1. Any collineation $\alpha$ of $a(v, k, \lambda)$ configuration permutes the points and the lines in such manner that there exists a one-to-one correspondence between cycles of points and cycles of lines with each pair of cycles of the same length. In particular $\alpha$ fixes equally many points and lines.

Proof. Number the points $p_{1}, \cdots, p_{v}$ and the lines $l_{1}, \cdots, l_{v}$ Let $A$ be the incidence matrix for the design defined by $A=\left(a_{i j}\right)$, $a_{i j}=1$ if $p_{i} \in l_{j}$ and 0 otherwise. $\alpha$ corresponds to a unique pair $(P, Q)$ of permutation matrices such that $P A=A Q$. $P$ and $Q$ permute the points and the lines respectively. $A$ is nonsingular. Thus $A^{-1} P A=Q$. $P$ and $Q$ being similar have the same set of characteristic roots with like multiplicities.

For each divisor $d$ of the order of the permutation $P$, each primitive $d$ th root of unity is a characteristic root of $P$ with multiplicity equal to the number of cycles of $P$ of length divisible by $d$. Counting characteristic roots, it follows that $P$ and $Q$ have equally many cycles of any length.

Using the above will be proved:

TheOREM 2. Any group $G$ of collineations of $a(v, k, \lambda)$ configuration has equally many transitive sets on the points and on the lines. (Note: a fixed point or line is counted as a transitive set.)

Proof. Let $g$ be the order of $G$. $G$ is represented by pairs $\left(P_{t}, Q_{t}\right)$, $t=1, \cdots, g$, of permutation matrices, $P_{t}$ and $Q_{t}$ on points and lines respectively. The total number of points fixed [2] by all the $P_{t}$ is $r g$, where $r$ is the number of transitive sets (counting fixed points) of

Received by the editors July 3, 1956.

1 This work was performed while under Army Ordnance Corps Contract No. DA-33-019-ORD-1911. 
$\left\{P_{t}\right\} \sim G$. Likewise the $Q_{t}$ fix $r^{\prime} g$ lines, $r^{\prime}$ the number of transitive sets of $\left\{Q_{t}\right\} \sim G$. By Theorem 1, each corresponding $P_{t}$ and $Q_{t}$ fix equally many points and lines. Summing over the elements of $G, r g=r^{\prime} g$, so that $G$ has equally many transitive sets of points and lines.

Added February 16, 1957. Another fairly immediate consequence is the following:

TheOREM 3. If $a(v, k, \lambda)$ configuration with incidence matrix $A$ has a collineation defined by $P A=A Q$, where $P$ and $Q$ are permutation matrices, then the configuration possesses an incidence matrix $A^{\prime}$ such that $P A^{\prime}=A^{\prime} P$.

Proof. By Theorem 1, the permutations defined by $P$ and $Q$ are similar, and hence conjugate in the symmetric permutation group of degree $v$. Thus there exists a permutation matrix $R$ such that $Q$ $=R^{-1} P R$. In turn, $P A=A\left(R^{-1} P R\right)$, so that $P\left(A R^{-1}\right)=\left(A R^{-1}\right) P$. $A R^{-1}$ is merely the incidence matrix $A$ with columns permuted; the $A^{\prime}$ asserted to exist is $A R^{-1}$.

Theorem 2 has been proved by entirely different methods by $D$. R. Hughes in a forthcoming paper in the Transactions of the American Mathematical Society.

\section{REFERENCES}

1. H. J. Ryser, Geometries and incidence matrices, Slaught Papers no. 4, Math. Assn. of America, 1955.

2. A. Speiser, Theorie der Gruppen von endlicher Ordnung, 3d ed., Dover, Theorem 102 , p. 118.

Ohio State University 